\title{
The use of nanocrystalline cellulose for the binding and controlled release of drugs
}

\author{
This article was published in the following Dove Press journal: \\ International Journal of Nanomedicine \\ 9 February 2011 \\ Number of times this article has been viewed
}

\author{
John K Jackson' \\ Kevin Letchford' \\ Benjamin Z Wasserman' \\ Lucy Ye' \\ Wadood Y Hamad ${ }^{2}$ \\ Helen M Burt' \\ 'Faculty of Pharmaceutical Sciences, \\ University of British Columbia, \\ 2I 46 East Mall,Vancouver, BC, Canada; \\ ${ }^{2}$ FPInnovations, 3800 Wesbrook Mall, \\ Vancouver, BC, Canada
}

Correspondence: Helen M Burt

2I 46 East Mall,Vancouver,

BCV6T IZ3, Canada

Tel +l 6048222440

Fax +l 6048223035

Email burt@interchange.ubc.ca
Abstract: The objective of this work was to investigate the use of nanocrystalline cellulose (NCC) as a drug delivery excipient. NCC crystallites, prepared by an acid hydrolysis method, were shown to have nanoscopic dimensions and exhibit a high degree of crystallinity. These crystallites bound significant quantities of the water soluble, ionizable drugs tetratcycline and doxorubicin, which were released rapidly over a 1-day period. Cetyl trimethylammonium bromide (CTAB) was bound to the surface of NCC and increased the zeta potential in a concentration-dependent manner from -55 to $0 \mathrm{mV}$. NCC crystallites with CTAB-modified surfaces bound significant quantities of the hydrophobic anticancer drugs docetaxel, paclitaxel, and etoposide. These drugs were released in a controlled manner over a 2-day period. The NCC-CTAB complexes were found to bind to KU-7 cells, and evidence of cellular uptake was observed.

Keywords: drug delivery, nanocrystalline cellulose, controlled release

\section{Introduction}

Cellulose has a long history of use in the pharmaceutical industry. The material has excellent compaction properties when blended with other pharmaceutical excipients so that drug-loaded tablets form dense matrices suitable for the oral administration of drugs. The form of cellulose used in tablets is termed microcrystalline cellulose (MCC) which is a purified, depolymerized alpha cellulose derived from plant sources. ${ }^{1}$ Despite an extended history of use in tableting, there is still considerable continuing research into the use of $\mathrm{MCC}$ and other types of cellulose in advanced pelleting systems whereby the rate of tablet disintegration and drug release may be controlled by microparticle inclusion, excipient layering or tablet coating. ${ }^{2-11}$

Derivatized cellulose has also been used extensively in pharmaceutical preparations so that ethyl cellulose, methyl cellulose, carboxymethyl cellulose, and numerous other forms are used in oral, topical, and injectable formulations. For example, carboxymethyl cellulose is the primary component of Seprafilm ${ }^{\mathrm{TM}}$, which is applied to surgical sites to prevent post surgical adhesions. Recently, the use of MCC in self-emulsifying drugdelivery systems and semisolid injectable formulations has also been described. ${ }^{2,12}$ The use of these forms of cellulose in such formulations points to the inertness and excellent biocompatibility of cellulose in humans. Additionally, hydroxypropyl methyl cellulose has recently been used as a hydrogel matrix for chondrocyte implantation into animal joints for cartilage repair. ${ }^{13}$

However, these uses of cellulose in formulations do not involve direct molecular level control of drug release via binding interactions with the drug. Although the surface of MCC has a slight negative charge due to hydroxyl residues, this charge is 
confined to a relatively small surface area on a large mass of insoluble MCC and would not likely adsorb or bind significant amounts of drug. The principle of using charged particles to bind acidic or basic drugs is well established as evidenced by the use of ion-exchange resins for the past 50 years to bind and release drugs. ${ }^{14}$ Drug release from resins is usually rapid once the resin-drug complex reaches the target site, since counter ions present in body fluids displace the drug from the binding site. For extended release, resins have been coated with agents such as ethyl or carboxymethyl cellulose to delay drug elution. Complex polysaccharides such as chitosan have been used extensively in controlled release drug formulations. Such methods rely on a charge interaction between the positive charge on amine groups of each sugar residue in chitosan with negatively charged drugs such as antisense oligonucleotides. ${ }^{15,16}$ The high positive and negative charges on chitosan and oligonucleotides respectively, allow for a strong binding interaction between the carrier and the drug so that phosphate counterions tend to release a weakly bound fraction rapidly and a tightly bound fraction very slowly. ${ }^{16}$

Nanocrystalline cellulose (NCC) is extracted from lignocellulosics by acidic extraction methods resulting in the formation of nanodimensional cellulose rods, termed crystallites, with a very high surface to volume ratio. ${ }^{17}$ Due to its large surface area, high aspect ratio, and tremendous stiffness and strength, NCC is actively being investigated as a nanocomposite material. For a thorough review of the preparation, properties, modification, and application of NCC, readers are directed to the excellent review by Habibi et al. ${ }^{18}$

NCC offers several potential advantages as a drug delivery excipient. The very large surface area and negative charge of NCC suggests that large amounts of drugs might be bound to the surface of this material with the potential for high payloads and optimal control of dosing. Other nanocrystalline materials, such as nanocrystalline clays have been shown to bind and subsequently release drugs in a controlled manner via ion exchange mechanisms and are being investigated for use in pharmaceutical formulations. ${ }^{19}$ The established biocompatibility of cellulose supports the use of NCC for a similar purpose. The abundant surface hydroxyl groups on NCC provide a site for the surface modification of the material with a range of chemical groups by a variety of methods. Surface modification may be used to modulate the loading and release of drugs that would not normally bind to NCC, such as nonionized or hydrophobic drugs. For example, Lonnberg et al suggested that poly(caprolactone) chains might be conjugated onto nanocrystalline cellulose for such a purpose. ${ }^{20}$ Additionally, since NCC is a low-cost, readily abundant material from a renewable and sustainable resource, its use provides a substantial environmental advantage compared with other nanomaterials.

The objectives of this work were to investigate the binding and release of a range of ionized drugs from NCC and to surface modify NCC with the surfactant, cetyl trimethylammonium bromide (CTAB) for the binding and release of hydrophobic drugs. To our knowledge, this is the first report of the use of NCC as a drug delivery excipient.

\section{Materials and methods \\ Materials}

Nanocrystalline cellulose was prepared according to the method outlined below by FPInnovations (Vancouver, BC, Canada). A $4 \mathrm{mg} / \mathrm{mL}$ suspension in deionized water was used for this investigation. Docetaxel was obtained from Natural Pharma Inc, (Vancouver, BC, Canada). Doxorubicin hydrochloride, paclitaxel, and etoposide were supplied by Polymed Therapeutics, Inc (Houston, TX, USA). Cetyl trimethylammonium bromide and tetracycline hydrochloride were supplied by Sigma Aldrich (Oakville, ON, Canada). Methoxy poly(ethylene glycol)-block-poly (D,L lactide) (MePEG-b-PDLLA) diblock copolymer was synthesized and characterized as previously described. ${ }^{21}$

\section{NCC preparation}

NCC was prepared by a previously reported method. ${ }^{22}$ Briefly, a fully bleached, commercial softwood was milled to pass through a $0.5-\mathrm{mm}$ screen in a Wiley mill to ensure particle size uniformity and increased surface area. The milled pulp (40.0 g) was hydrolyzed in $64 \% \mathrm{w} / \mathrm{w}$ sulfuric acid $(8.75 \mathrm{~mL}$ of a sulfuric acid solution/g pulp) at $45^{\circ} \mathrm{C}$ and stirred at high speed for 25 minutes. The cellulose suspension was then diluted with cold, deionized distilled water $\left(\mathrm{dH}_{2} \mathrm{O}\right)$ and allowed to settle overnight. The clear top layer was decanted and the remaining white cloudy layer was washed with deionized water and centrifuged three times to remove practically all soluble cellulose materials. The resulting thick, white suspension was dialyzed against slow running $\mathrm{dH}_{2} \mathrm{O}$ for $1-4$ days using dialysis tubing with a 12,000-14,000 molecular weight cutoff (Spectra/Por ${ }^{\circledR}$, Spectrum Laboratories, Inc, Rancho Dominguez, CA, USA). The suspension from the dialysis tubes was dispersed by subjecting it to ultrasound treatment in a Fisher Sonic Dismembrator (Fisher Scientific, Ottawa, ON, Canada) for 10 minutes at $60 \%$ power.

\section{X-ray powder diffraction}

Diffraction data were collected on films of lyophilized and air dried NCC using a previously described method. ${ }^{23}$ 
Briefly, a Bruker D8 Advance powder x-ray diffractometer (Bruker Corporation, Milton, ON, Canada) equipped with a $\mathrm{CuX} \mathrm{x}_{\alpha}$-ray tube, a diffracted beam graphite monochromator, and Nal scintillation detector was used with the generator set to $40 \mathrm{kV}$ and $40 \mathrm{~mA}$. Data was collected from $5^{\circ}-90^{\circ}$ $2 \theta$ using a step size of $0.02^{\circ}$ and a counting time of $1 \mathrm{sec} / \mathrm{step}$. A divergence of $1.00 \mathrm{~mm}$ and anti-scatter slits were used with a $0.2 \mathrm{~mm}$ receiving slit, and the sample was rotated during data collection. The films were mounted on zero-background Si plates. The peaks were assigned according to the monoclinic unit cell for cellulose I. The crystallinity was obtained from the X-ray intensity, as described by Hamad and $\mathrm{Hu} .^{23}$

\section{Imaging}

Air-dried films or a drop of the NCC suspension in $\mathrm{dH}_{2} \mathrm{O}$ were mounted on aluminum stubs using adhesive carbon tabs, and the stubs were sputter-coated with AuPd. Morphological characteristics were observed using an FEI Quanta 400F field-emission scanning electron microscope (SEM) (FEI Company, Hillsboro, OR, USA), operated in high vacuum mode with a $5 \mathrm{kV}$ accelerating voltage.

\section{NCC drug binding}

Doxorubicin hydrochloride (DOX) or tetracycline hydrochloride (TET) were dissolved in either $10 \mathrm{mM}$ phosphate buffered saline (PBS) at $\mathrm{pH} 7.4$, or $\mathrm{dH}_{2} \mathrm{O}$ with increasing drug concentrations $\left(\left[\operatorname{drug}_{\text {added }}\right]\right)$. Drug solutions $(1.5 \mathrm{~mL})$ were added to $0.5 \mathrm{~mL}$ of a $4 \mathrm{mg} / \mathrm{mL}$ NCC suspension (2 $\mathrm{mg}$ of $\mathrm{NCC}$ ) in a $2 \mathrm{~mL}$ microcentrifuge tube and incubated at $37^{\circ} \mathrm{C}$ with tumbling at $8 \mathrm{rpm}$ for 30 minutes. Suspensions containing PBS or $\mathrm{NaCl}$ produced flocculated $\mathrm{NCC} /$ drug suspensions, which were centrifuged at $18000 \times g$ for 10 minutes to pellet the NCC and bound drug. The concentration of unbound drug in the supernatant ([drug $\left.\left.{ }_{\text {unbound }}\right]\right)$ was assayed using a Varian 50 Bio UV Vis spectrophotometer (Varian, Inc., Mississauga, ON, Canada) using wavelengths of $482 \mathrm{~nm}$ and $364 \mathrm{~nm}$ for DOX and TET, respectively. The concentration of drug bound to the NCC ([ $\left.\operatorname{drug}_{\text {bound }}\right]$ ) was calculated using the following formula:

$$
\left[\operatorname{drug}_{\text {bound }}\right]=\left[\operatorname{drug}_{\text {added }}\right]-\left[\operatorname{drug}_{\text {unbound }}\right] .
$$

NCC does not flocculate in $\mathrm{dH}_{2} \mathrm{O}$; therefore, the $\mathrm{NCC} /$ drug complexes prepared in $\mathrm{dH}_{2} \mathrm{O}$ could not be separated by microcentrifugation. In this case, the $\mathrm{NCC} /$ drug suspensions were transferred to dialysis bags with a molecular weight cutoff of 10,000 Da (Spectra/Por ${ }^{\circledR}$, Spectrum Laboratories, Inc., Rancho Dominguez, CA, USA) and dialyzed against $\mathrm{dH}_{2} \mathrm{O}$ overnight in the dark at $4^{\circ} \mathrm{C}$. The concentration of unbound drug in the dialysate was determined by UV Vis spectroscopy, allowing for the calculation of the amount of drug bound to the NCC according to Equation 1.

To solubilize the hydrophobic drugs paclitaxel (PTX), docetaxel (DTX), and etoposide (ETOP), the surface of the NCC was first modified with CTAB. This was achieved by incubating increasing amounts of CTAB with $2.5 \mathrm{mg}$ of NCC so the final CTAB concentration varied from $0 \mathrm{mM}$ to $12.9 \mathrm{mM}$. An aliquot of $100 \mathrm{mM} \mathrm{NaCl}$ was added, resulting in a final $\mathrm{NaCl}$ concentration of $10 \mathrm{mM}$, which facilitated flocculation and subsequent separation of the NCC/CTAB nanocomplexes by centrifugation as described above. The NCC/CTAB was incubated with stock solutions of the drugs with increasing concentrations. Since these drugs are characterized by low aqueous solubility, they were solubilized in a minimal amount of either DMSO or MePEG-b-PDLLA diblock copolymer in $10 \mathrm{mM} \mathrm{NaCl}$ as previously described. ${ }^{21}$ The drug/NCC/CTAB suspensions were incubated at $25^{\circ} \mathrm{C}$ with tumbling at $8 \mathrm{rpm}$ for 30 minutes then centrifuged at $18000 \times g$ for 10 minutes to pellet the $\mathrm{NCC} / \mathrm{CTAB}$ and bound drug. The amount of unbound drug in the supernatant was determined by high performance liquid chromatography (HPLC) using a Waters HPLC system with Millennium software and UV Vis detection (Waters Ltd, Mississauga, ON, Canada). Separation was achieved using a Novapak C18 column with $20 \mu \mathrm{L}$ injections and a mobile phase flow rate of $1 \mathrm{~mL} / \mathrm{min}$. The DTX and PTX mobile phase consisted of $58 \%$ acetonitrile, $37 \% \mathrm{dH}_{2} \mathrm{O}$, and $5 \%$ methanol, with detection at $232 \mathrm{~nm}$. The mobile phase for ETOP was $27 \%$ acetonitrile, $1 \%$ acetic acid, and $72 \%$ $\mathrm{dH}_{2} \mathrm{O}$, and detection was at $286 \mathrm{~nm}$. Calibration curves were prepared for all drugs and were linear in the desired concentration range. The amount of drug bound to the NCC/CTAB was determined using Equation 1.

\section{Drug release studies}

DOX was bound to NCC for release studies by incubating a solution of $325 \mu \mathrm{g} / \mathrm{mL}$ of DOX in $\mathrm{dH}_{2} \mathrm{O}$ with a suspension containing $2.5 \mathrm{mg}$ of NCC. To flocculate the NCC and allow for separation of the NCC/DOX nanocomplex, $\mathrm{NaCl}$ was added to a final concentration of $10 \mathrm{mM}$. The suspension was centrifuged at $18000 \times g$ for 10 minutes to pellet the $\mathrm{NCC} /$ DOX, and the drug binding was determined by UV Vis spectroscopy as described above. The final mass of DOX bound to the NCC for the release studies was $212 \pm 3.5 \mu \mathrm{g}$. The same procedure was used to prepare TET-bound NCC nanocomplexes for the release studies, with the exception that the initial TET solution used was $1000 \mu \mathrm{g} / \mathrm{mL}$, which resulted in the 
binding of $187 \pm 2.0 \mu \mathrm{g}$ of TET. NCC/drug nanocomplexes with DTX, PTX, and ETOP were prepared as described for the drug-binding studies. The concentration of DTX and PTX that was incubated with the NCC suspension was $200 \mu \mathrm{g} / \mathrm{mL}$, and the concentration of ETOP was $100 \mu \mathrm{g} / \mathrm{mL}$. The final mass of drug bound to the NCC was $184 \pm 4.8 \mu \mathrm{g}, 149 \pm 4.8 \mu \mathrm{g}$, and $63 \pm 0.1 \mu \mathrm{g}$ for DTX, PTX, and ETOP, respectively. The drug loaded NCC samples were resuspended in $1 \mathrm{~mL}$ of PBS followed by incubation at $37^{\circ} \mathrm{C}$ with tumbling at $8 \mathrm{rpm}$. At predetermined times, the suspensions were centrifuged at $18000 \times g$ for 10 minutes, and the supernatant was removed for drug quantification by UV Vis for DOX and TET or HPLC for DTX, PTX, and ETOP, as previously described. At each sampling time point, fresh PBS was added to the tubes, and the NCC/drug nanocomplexes were resuspended.

\section{Zeta potential measurements}

The effect of CTAB concentration on zeta potential of NCC was determined by incubating $2.5 \mathrm{mg}$ samples of NCC with increasing concentrations of CTAB in $\mathrm{dH}_{2} \mathrm{O}$ to yield final CTAB concentrations of $0,0.755,1.13,1.51,1.89,2.27$, and $3.02 \mathrm{mM}$. The zeta potentials of the samples were measured using a Malvern 3000 HS-Zetasizer (Malvern Instruments Ltd, Malvern, UK).

\section{Cell binding and uptake studies}

KU-7 bladder cancer cells were a kind gift from Dr Martin Gleave at the Prostate Centre, Vancouver General Hospital and were grown in Dulbecco's Modified Eagle's Medium supplemented with 10\% fetal bovine serum, 5\% glutamine, and $5 \%$ penicillin/streptomycin. Cells were plated at 10,000 cells per well on a 96-well cell culture plate and allowed to equilibrate overnight. NCC for binding studies was prepared by coating with CTAB followed by fluorescein binding. Briefly, $2 \mathrm{mg}$ of NCC were suspended in a $3 \mathrm{mM}$ CTAB in $5 \mathrm{mM}$ sodium chloride solution for 30 minutes at $37^{\circ} \mathrm{C}$. The suspension was centrifuged at $18,000 \times g$ for 30 seconds and the supernatant discarded. The pellet was resuspended in $1 \mathrm{~mL}$ of a $5 \mathrm{mM}$ sodium chloride solution containing $100 \mu \mathrm{g} / \mathrm{mL}$ fluorescein and incubated for 30 minutes at $37^{\circ} \mathrm{C}$. The suspension was centrifuged at $18,000 \times g$ for 30 seconds, and the supernatant was discarded followed by resuspension in $\mathrm{PBS}$ and serial dilution to give concentrations ranging from 10 to $0.06 \mathrm{mg} / \mathrm{mL}$. To each cell culture plate well, $200 \mu \mathrm{L}$ of each NCC suspension was added, and the cells were allowed to incubate for 24 hours. The media was removed and the cells washed three times with PBS prior to lysis with $2 \%$ Triton X-100. The fluorescence intensity of the
$\mathrm{NCC} / \mathrm{CTAB} /$ fluorescein bound to the cells was determined using a fluorescence spectroscopy plate reader with excitation and emission wavelengths of 480 and $520 \mathrm{~nm}$, respectively. These values were converted to mass of NCC/CTAB/ fluorescein bound to cells using a previously constructed standard curve of fluorescence intensity as a function of mass of NCC/CTAB/fluorescein. A BCA total protein assay kit (Pierce Chemicals, Rockford, IL, USA) was used to ensure that each well contained the same number of cells.

To visualize cell uptake of NCC, KU-7 cells were cultured as described for the binding studies. Glass cover slips ( $1 \mathrm{~cm}$ diameter) were sterilized and placed in the base of 6 well tissue culture plates followed by the addition of 50,000 cells to each well and incubation overnight. Cells were incubated with a $1.25 \mathrm{mg} / \mathrm{mL}$ NCC/CTAB/ fluorescein suspension for various times at $37^{\circ} \mathrm{C}$ under culture conditions. The cover slips were removed, washed three times in PBS and then treated with 4',6-diamidino-2phenylindole (DAPI) in Progold stain for 30 minutes, washed again and then treated with $3.7 \%$ paraformaldehyde in $\mathrm{dH}_{2} \mathrm{O}$ for 30 minutes. The cover slips were placed cell side down on microscope slides and sealed with clear nail varnish. Fluorescein- and DAPI-stained nuclei were visualized using an Olympus FV-1000 confocal laser scanning microscope (Olympus Canada, Inc., Markham, ON, Canada).

\section{Results}

\section{X-ray powder diffraction} and imaging of NCC

The X-ray powder diffraction (XRPD) pattern of NCC was characterized by two major peaks centered around $15^{\circ}$ and $23^{\circ} 2 \theta$ (Figure 1). The degree of crystallinity of the sample was calculated to be greater than $90 \%$. The SEM images of NCC as a dried film show a layered pattern of NCC crystallites oriented in a common direction (Figure 2A).

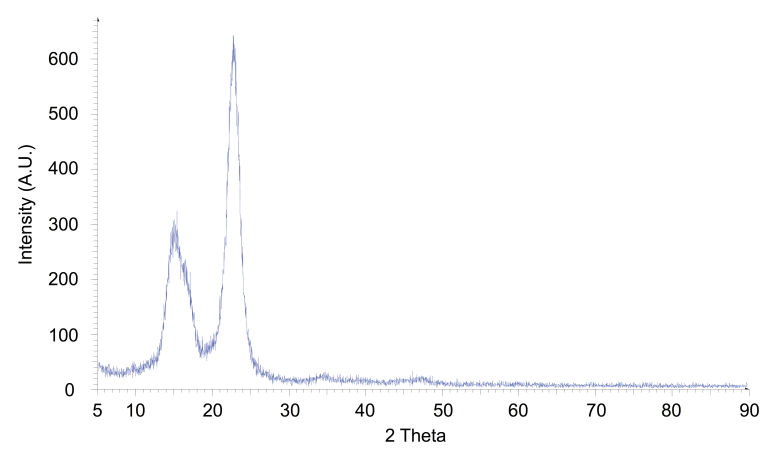

Figure I X-ray powder diffraction pattern of lyophilized nanocrystalline cellulose. Intense peaks at $15^{\circ}$ and $23^{\circ} 2 \theta$, due to constructive interference of diffracted $\mathrm{x}$-rays, in addition to the lack of an amorphous halo, indicate the highly crystalline nature of the material. 


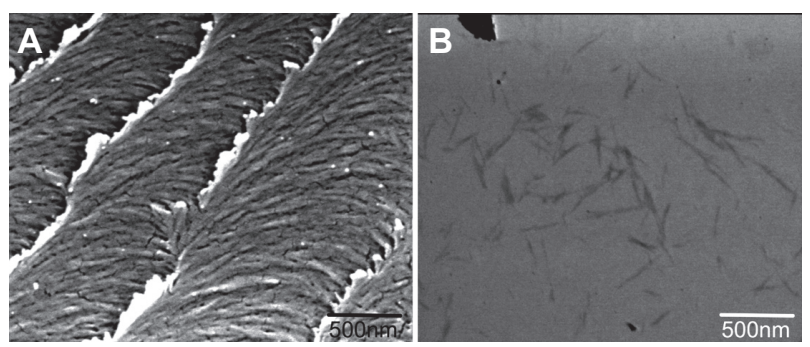

Figure 2 Scanning electron micrograph of a dried film of nanocrystalline cellulose (NCC) (A) and NCC crystallites in deionized distilled water (B).

The scanning transmission electron microscopy (STEM) micrograph of the NCC suspension shows individual NCC fibers with approximate dimensions of $10 \mathrm{~nm}$ wide by $500 \mathrm{~nm}$ in length (Figure 2B).

\section{Drug binding to NCC}

The amount of DOX bound to NCC increased significantly as the mass of drug added to the NCC suspension increased. When the dispersion medium was PBS, a maximum of $122 \mu \mathrm{g}$ of DOX was bound to NCC representing a $65 \%$ binding efficiency (Figure $3 \mathrm{~A}$ ). When $\mathrm{dH}_{2} \mathrm{O}$ was used as the dispersion medium, a maximum of $1667 \mu \mathrm{g}$ of DOX
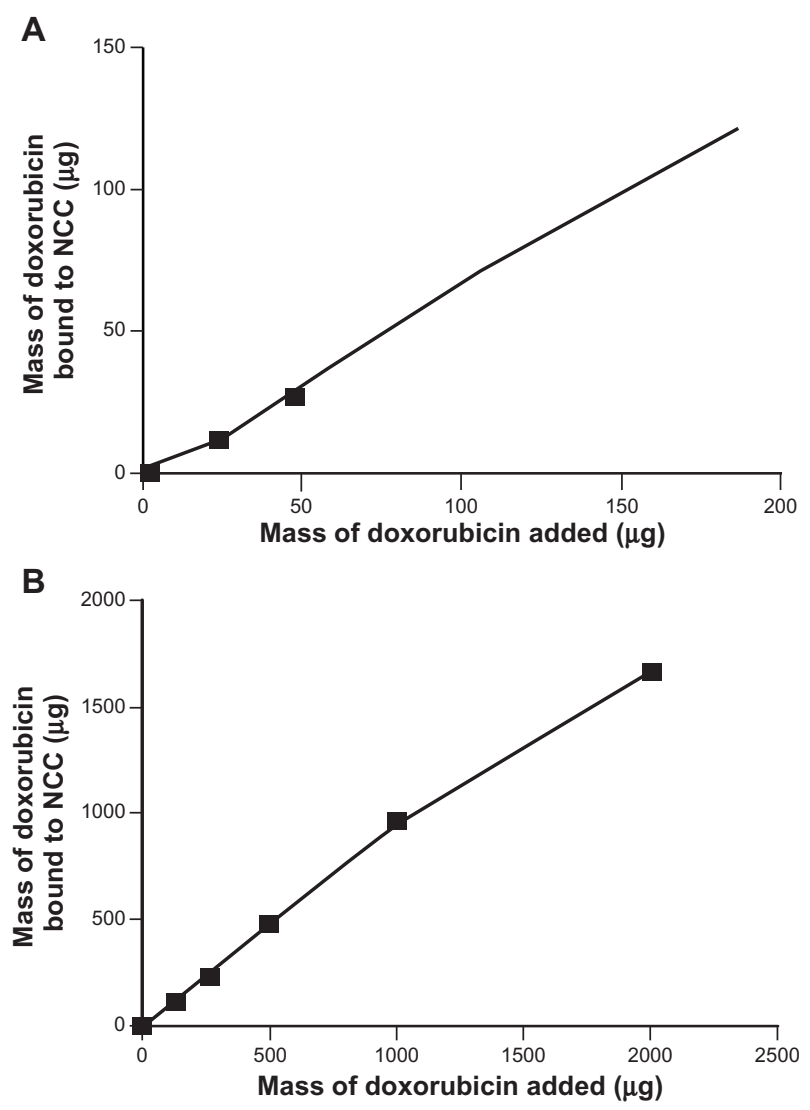

Figure 3 The binding of doxorubicin to $2 \mathrm{mg} \mathrm{NCC}$ in $10 \mathrm{mM}, \mathrm{pH} 7.4$ phosphate buffered saline at $25^{\circ} \mathrm{C}(\mathbf{A})$ and deionized distilled water at $25^{\circ} \mathrm{C}(\mathbf{B})$. Abbreviation: NCC, nanocrystalline cellulose. was bound to NCC with a binding efficiency of $83 \%$ (Figure 3B). It was found that the binding efficiency of TET to NCC was considerably less than that of DOX, regardless of the dispersion medium used (Figure 4). Using PBS as a dispersion medium, a maximum of $251 \mu \mathrm{g}$ of TET was bound with a $25 \%$ binding efficiency (Figure $4 \mathrm{~A}$ ). When the dispersion medium was $\mathrm{dH}_{2} \mathrm{O}, 959 \mu \mathrm{g}$ of TET was bound to NCC with a $48 \%$ binding efficiency (Figure 4B).

The effect of increasing concentration of CTAB coating on NCC on the binding of the hydrophobic drugs DTX, PTX, and ETOP was investigated (Figures 5-7). In all cases it was found that increased amounts of $\mathrm{CTAB}$ resulted in increased drug binding. At the highest CTAB concentration $(12.9 \mathrm{mM})$, the binding efficiency of DTX and PTX to the NCC/CTAB nanocomplexes was approximately $90 \%$ up to $100 \mu \mathrm{g}$ of drug added (Figures 5A and 6A). Above this drug concentration, the drug-binding efficiency decreased with saturation of binding occurring at approximately $200 \mu \mathrm{g}$ (Figure 5B and $6 \mathrm{~B})$. Much less ETOP was capable of binding to the NCC/CTAB nanocomplexes with a $48 \%$ binding efficiency and a maximum of $48 \mu \mathrm{g}$ bound when $100 \mu \mathrm{g}$ of drug was added to the NCC/CTAB (Figure 7).
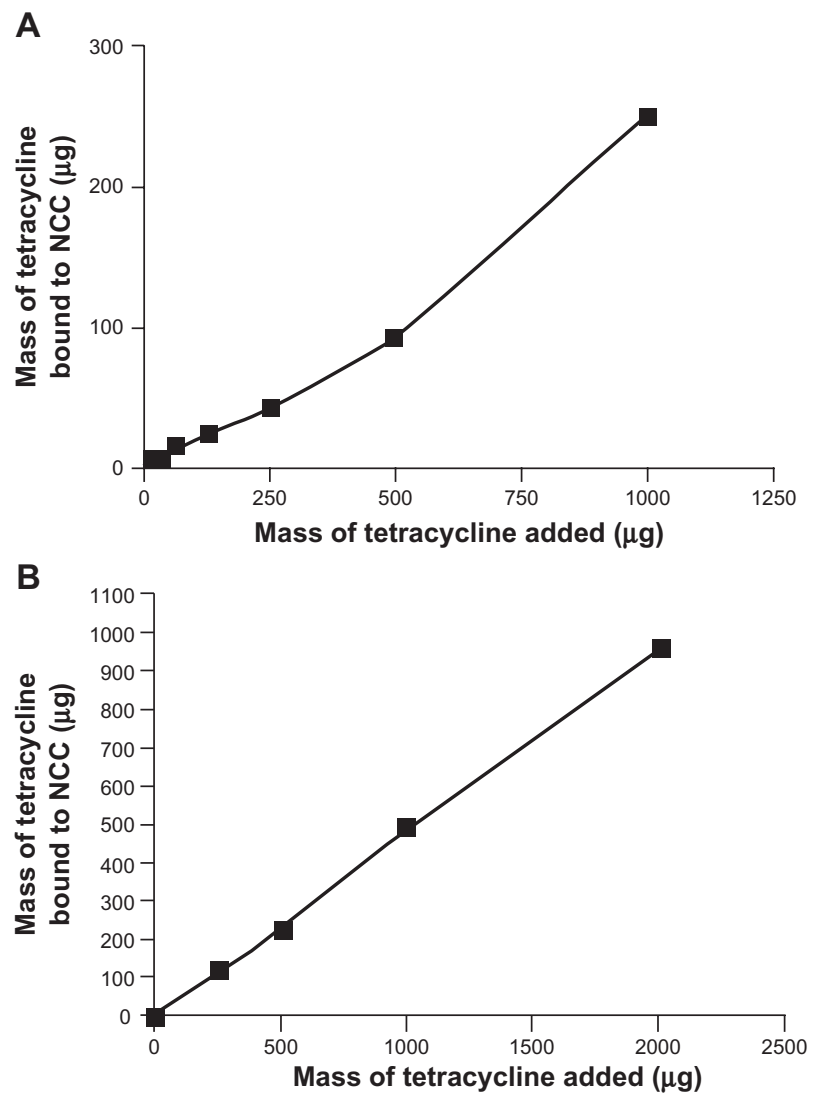

Figure 4 The binding of tetracycline to $2 \mathrm{mg} \mathrm{NCC}$ in $10 \mathrm{mM}$, pH 7.4 phosphate buffered saline at $25^{\circ} \mathrm{C}(\mathbf{A})$ or deionized distilled water at $25^{\circ} \mathrm{C}(\mathbf{B})$. Abbreviation: NCC, nanocrystalline cellulose. 

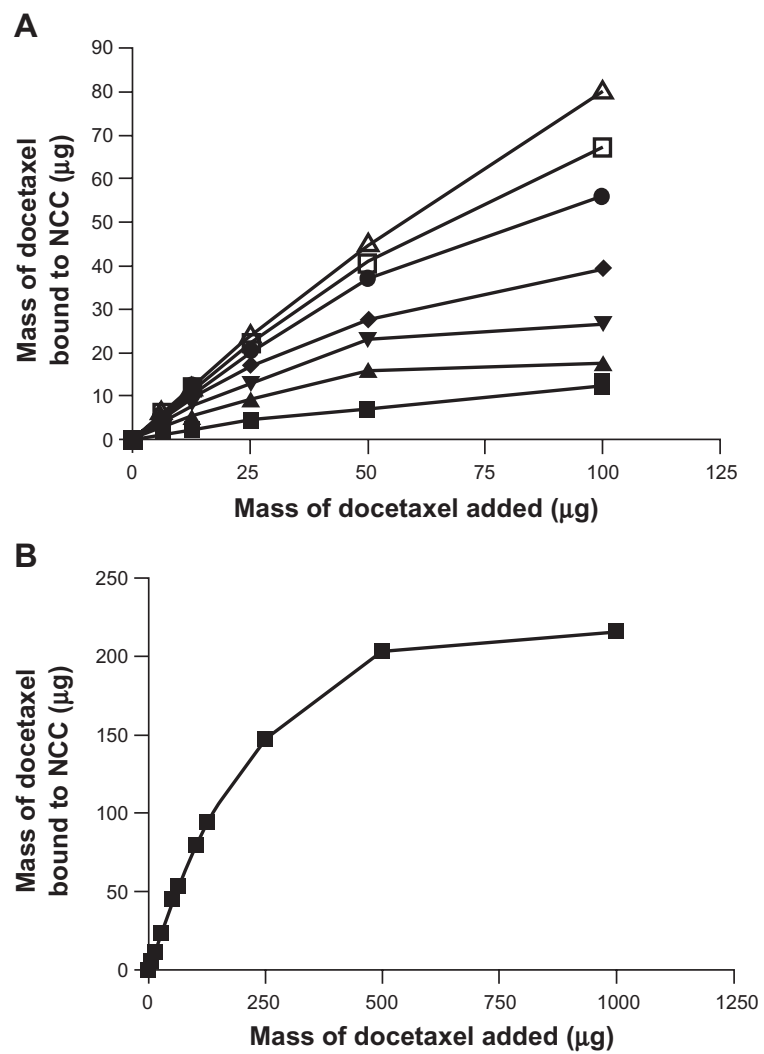

Figure $5 \mathrm{~A}$ ) The binding of docetaxel to $2.5 \mathrm{mg}$ of NCC/CTAB nanocomplexes in $10 \mathrm{mM} \mathrm{NaCl}$ at $25^{\circ} \mathrm{C}$ with $\mathrm{CTAB}$ concentrations of $0 \mathrm{mM}(\mathbf{\square}), 0.755 \mathrm{mM}$ $(\Delta), 1.5 \mathrm{I} \mathrm{mM}(\boldsymbol{\nabla}), 2.27 \mathrm{mM}(\bullet), 4.53 \mathrm{mM}(\bullet), 6.79 \mathrm{mM}(\square)$, and $12.9 \mathrm{mM}(\Delta)$. B) Maximal binding of docetaxel at a CTAB concentration of $12.9 \mathrm{mM}$.

Abbreviations: CTAB, cetyl trimethylammonium bromide; NCC, nanocrystalline cellulose.

\section{Release of drugs from NCC}

Both DOX and TET released rapidly from NCC, resulting in approximately $90 \%$ of bound TET and $85 \%$ of bound DOX released in 4 hours (Figure 8). By day 1, the drug release had plateaued, with $93 \%$ and $87 \%$ of TET and DOX released, respectively. The release profiles of the hydrophobic drugs DTX, PTX, and ETOP bound to NCC/CTAB are shown in Figure 9 . Approximately $26 \%$ of DTX was released within the first hour, followed by a slower but sustained release. In total, $59 \%$ of the total bound DTX was released in 2 days. A similar release profile was observed for PTX, which was characterized by a rapid release of $20 \%$ of bound drug in the first hour followed by slower release resulting in 44\% drug release over 2 days. The release of ETOP was similar to DTX and PTX, with the exception that a total of $75 \%$ of the drug was released over 4 days.

\section{$\mathrm{NCC} / \mathrm{CTAB}$ characterization}

In $\mathrm{dH}_{2} \mathrm{O}$, NCC remained as a stable colloidal dispersion and did not flocculate or sediment under high-speed
A

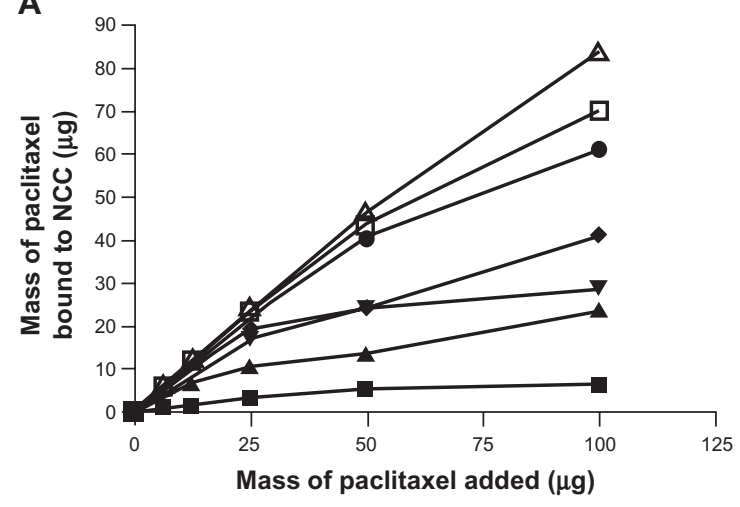

B

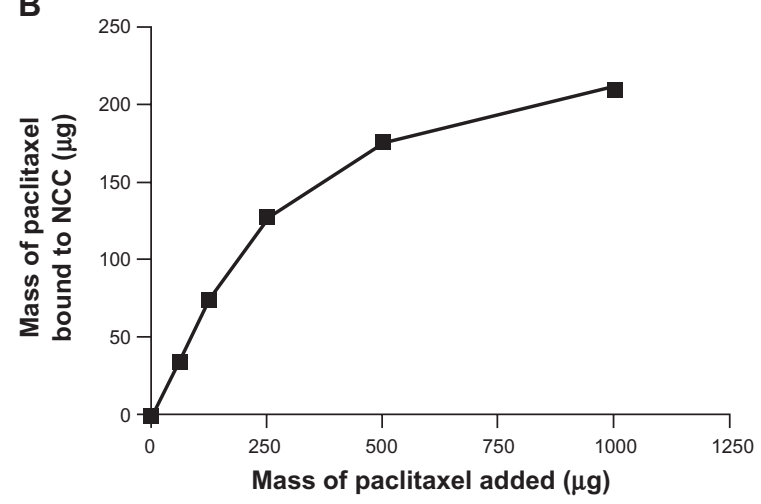

Figure $6 \mathbf{A}$ ) The binding of paclitaxel to $2.5 \mathrm{mg}$ of NCC/CTAB nanocomplexes in $10 \mathrm{mM} \mathrm{NaCl}$ at $25^{\circ} \mathrm{C}$ with $\mathrm{CTAB}$ concentrations of $0 \mathrm{mM}(\mathbf{\square}), 0.755 \mathrm{mM}(\mathbf{\Delta})$, I.5I mM ( $\nabla), 2.27 \mathrm{mM}(\bullet), 4.53 \mathrm{mM}(\bullet), 6.79 \mathrm{mM}(\square)$, and I $2.9 \mathrm{mM}(\Delta)$. B) Maximal binding of paclitaxel at a CTAB concentration of $12.9 \mathrm{mM}$.

Abbreviations: $C T A B$, cetyl trimethylammonium bromide; NCC, nanocrystalline cellulose.

centrifugation. However, when $5 \mathrm{mM}$ of $\mathrm{NaCl}$ was added, flocculation and subsequent sedimentation by high-speed centrifugation could be achieved. In water, CTAB had the same effect as $\mathrm{NaCl}$ so that at approximately $2 \mathrm{mM} \mathrm{CTAB}$, the NCC could be sedimented under centrifugation. At lower

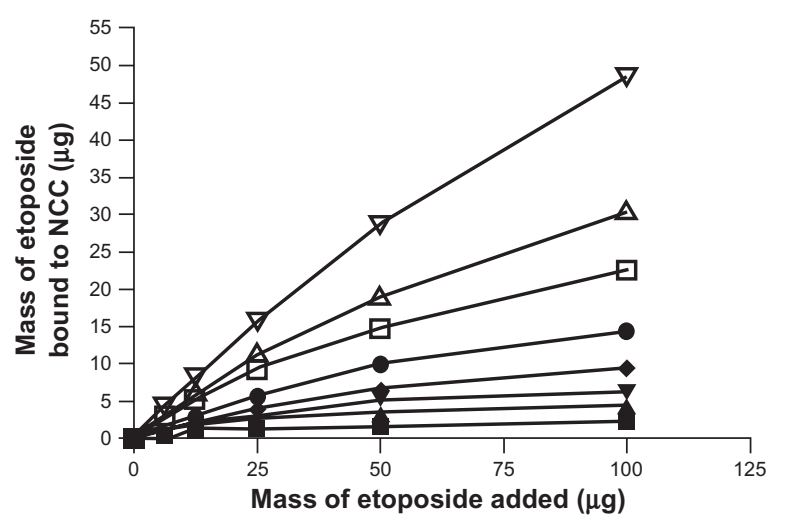

Figure 7 The binding of etoposide to $2.5 \mathrm{mg}$ of NCC/CTAB nanocomplexes in $10 \mathrm{mM} \mathrm{NaCl}$ at $25^{\circ} \mathrm{C}$ with $\mathrm{CTAB}$ concentrations of $0 \mathrm{mM}(\boldsymbol{\nabla}), 0.375 \mathrm{mM}(\boldsymbol{\Delta})$, $0.755 \mathrm{mM}(\boldsymbol{\nabla}), 1.5 \mathrm{I} \mathrm{mM}(\bullet), 2.27 \mathrm{mM}(\bullet), 4.53 \mathrm{mM}(\square)$, and $6.79 \mathrm{mM}(\Delta)$, and $12.9(\nabla)$.

Abbreviations: $C T A B$, cetyl trimethylammonium bromide; NCC, nanocrystalline cellulose. 


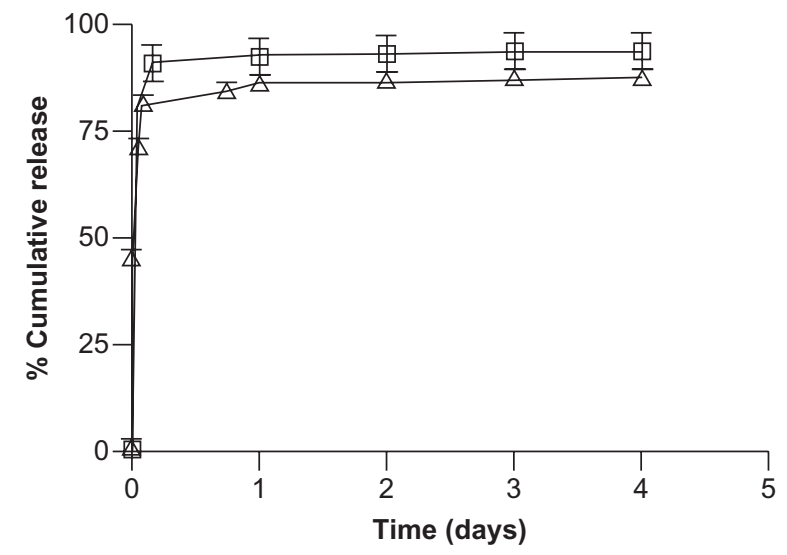

Figure 8 The in vitro release of doxorubicin $(\Delta)$ and tetracycline $(\square)$ from nanocrystalline cellulose in $10 \mathrm{mM}$ phosphate buffered saline at $37^{\circ} \mathrm{C}$.

concentrations of CTAB, a small amount of $\mathrm{NaCl}(10 \mathrm{mM})$ was added to tubes to enable sedimentation.

NCC had a strongly negative charge in water as evidenced by a zeta potential of approximately $-55 \mathrm{mV}$. Upon incubation with CTAB, the zeta potential increased in a concentration-dependent manner. At a concentration of $3 \mathrm{mM}$ CTAB, there was complete neutralization of the negative zeta potential (Figure 10).

\section{Cell binding and uptake studies}

More than $95 \%$ of the fluorescein bound to NCC/CTAB remained bound upon resuspension or dilution in aqueous media. When NCC/CTAB/fluorescein was incubated with KU-7 cells, the fluorescence signal was not quantifiable below $0.3 \mathrm{mg} / \mathrm{mL}$; however, above this concentration, the fluorescence increased in a concentration-dependent manner (Figure 11). At NCC/CTAB/fluorescein concentrations

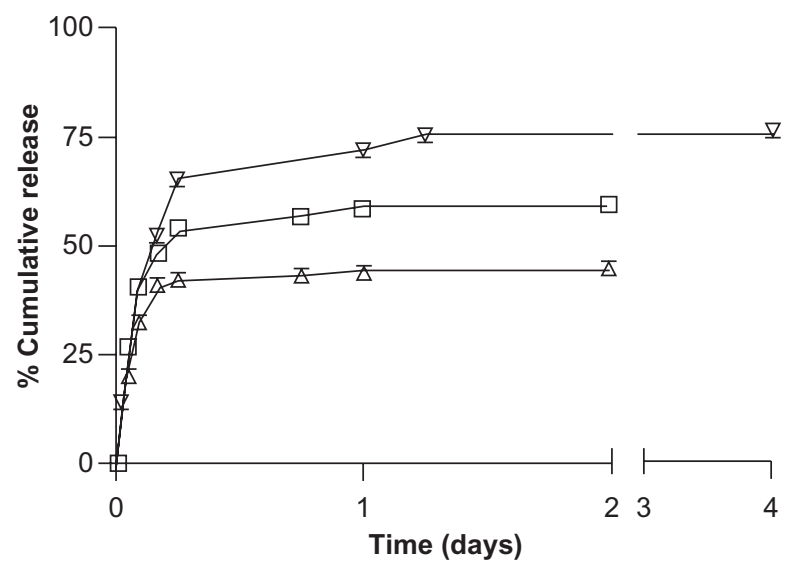

Figure 9 The in vitro release of etoposide $(\nabla)$, docetaxel $(\square)$, and paclitaxel $(\Delta)$ from NCC/CTAB nanocomplexes with $12.9 \mathrm{mM} C T A B$ in $10 \mathrm{mM}$ phosphate buffered saline at $37^{\circ} \mathrm{C}$.

Abbreviations: $C T A B$, cetyl trimethylammonium bromide; NCC, nanocrystalline cellulose.

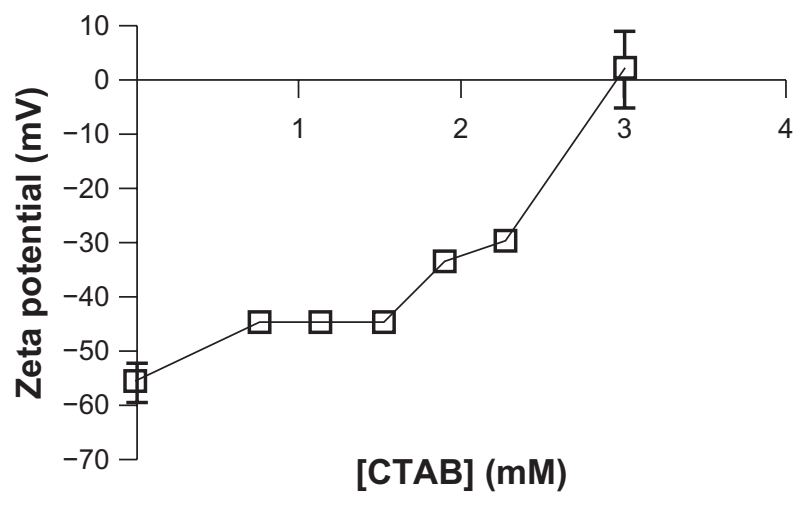

Figure 10 Zeta potential of NCC/CTAB nanocomplexes as a function of CTAB concentration.

Abbreviations: CTAB, cetyl trimethylammonium bromide; NCC, nanocrystalline cellulose.

greater than $1.25 \mathrm{mg} / \mathrm{mL}$, there was no linearity of the fluorescein quantitation, and it was not possible to accurately measure fluorescein uptake or binding to the cells.

The nuclei of the KU-7 cells displayed pronounced staining with DAPI as shown in Figure 12B. There is clear evidence of cellular uptake of fluorescein as demonstrated by strong fluorescence emission from the cytoplasm of the cells (Figure 12C). The uptake of fluorescein reached a maximum by 2 hours with little increase in cytoplasmic fluorescence emission after longer incubations. Cell uptake was observed using $\mathrm{NCC} / \mathrm{CTAB} /$ fluorescein nanocomplex concentrations of $0.25,0.50$, and $1.00 \mathrm{mg} / \mathrm{mL}$. There was no evidence of cell lysis with these complexes for up to 24 hours.

\section{Discussion}

Due its large surface area, stiffness, strength, and phase behavior, NCC is actively being investigated as a material

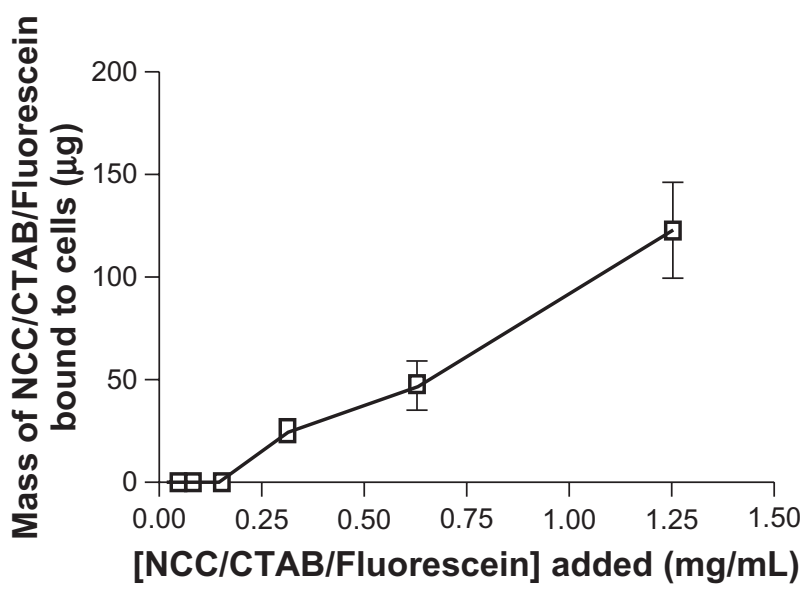

Figure I I Mass of $\mathrm{NCC} / \mathrm{CTAB} /$ fluorescein bound to $\mathrm{KU}-7$ cells as a function of concentration of NCC/CTAB/fluorescein added to cells.

Abbreviations: $C T A B$, cetyl trimethylammonium bromide; NCC, nanocrystalline cellulose. 

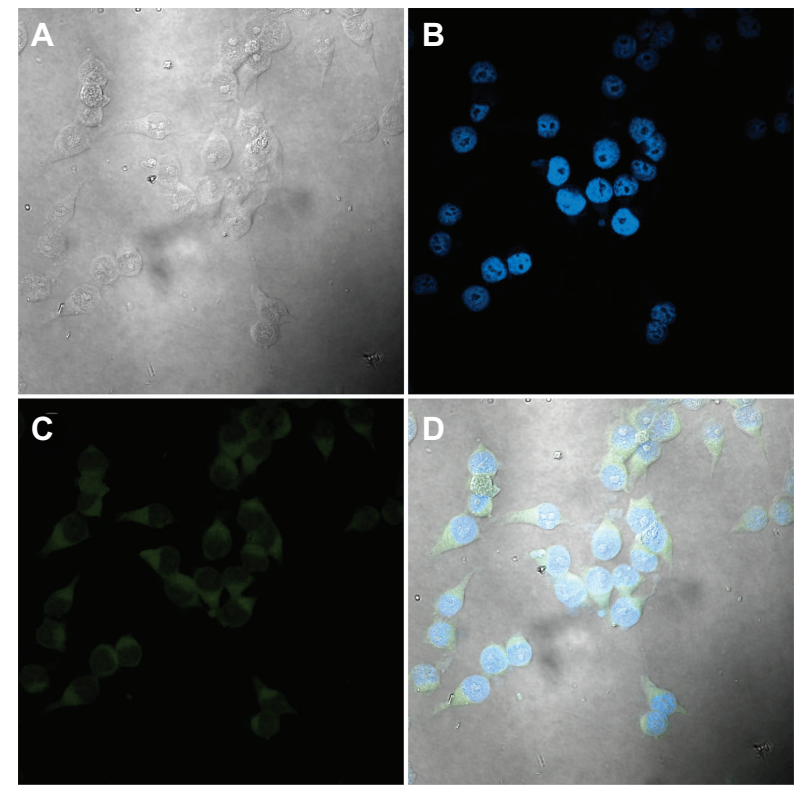

Figure 12 Confocal micrographs of KU-7 cells incubated for 2 hours with $\mathrm{NCC} / \mathrm{CTAB} /$ fluorescein nanocomplexes with a NCC/CTAB/fluorescein concentration of $0.25 \mathrm{mg} / \mathrm{mL}$. A) White light image of KU-7 cells. B) Staining of the nuclei with DAPI. C) Fluorescein in the cytoplasm. D) An overlay of images B and C.

Abbreviations: CTAB, cetyl trimethylammonium bromide; DAPI, 4',6-diamidino2-phenylindole; NCC, nanocrystalline cellulose.

for use in the packaging, aerospace, automotive, and cosmetic industries. However, to our knowledge, this is the first report of the use of NCC as an excipient for drug binding and subsequent controlled release. The high surface charge of NCC and the established biocompatibility of cellulosic materials suggest significant opportunities for exploration of the potential pharmaceutical applications of NCC. Other workers have described the use of chitosan, a positively charged insoluble polysaccharide, as a binding agent for the controlled release of negatively charged antisense oligonucleotide (ASO) drugs. ${ }^{15,16}$ In such studies, the chitosan/ASO complexes were used either alone or dispersed in a polymeric vehicle for the treatment of cancer.

Using an acid hydrolysis method, cellulose crystallites, or what we termed NCC crystallites, were isolated and investigated for use as a drug delivery excipient. The XRPD pattern, which is characterized by two high intensity peaks, indicates the high degree of crystallinity of the material (Figure 1). Additionally, the SEM images of these fibers clearly demonstrate the nanoscopic dimensions of this material when in a $\mathrm{dH}_{2} \mathrm{O}$ suspension (Figure 2B) and, as reviewed by Hamad, ${ }^{17}$ the ability of the material to yield a chiral nematic phase when dried as a film (Figure 2A).

These studies have demonstrated that hydrophilic drugs may bind directly to the surface of NCC at relatively high weight ratios (Figures 3 and 4) (eg, almost $500 \mu \mathrm{g}$ of TET may be bound to just $2 \mathrm{mg}$ of NCC, offering a $20 \% \mathrm{w} / \mathrm{w}$ drug loading (Figure 4)). The two hydrophilic drugs studied (TET and DOX) probably bound by an ionic interaction with the negatively charged surface of NCC since DOX is a cationic species slightly positively charged and TET is zwitterionic. Interestingly, considerably less DOX bound to $\mathrm{NCC}$ when dispersed in PBS as compared with $\mathrm{dH}_{2} \mathrm{O}$. This may be attributed to interference with DOX binding due to the presence of large amounts of counterions in PBS. Both these agents released rapidly from NCC in vitro (Figure 8), likely due to PBS counterions displacing the drugs via ion exchange. Such rapid release profiles are also seen for acidic or basic drugs bound to ion-exchange resins. ${ }^{14}$ Nevertheless, these rapid-release profiles observed for NCC may be suitable for potential applications as wound-dressing materials or for implantation into surgical resection voids such as tumor removal sites or periodontal cavities.

This work also demonstrates that NCC can be surface modified to deliver hydrophobic antiproliferative drugs. By coating the negatively charged NCC with a cationic surfactant (CTAB) it was possible to create a hydrophobic domain on the surface of the NCC. A clear interaction between $\mathrm{CTAB}$ and NCC was observed by flocculation phenomena at higher $\mathrm{CTAB}$ concentrations. Furthermore, the zeta potential (surface charge) on the NCC became increasingly less negative as the concentration of $\mathrm{CTAB}$ increased, providing evidence of a binding interaction between the CTAB and the NCC (Figure 10). The strong association between NCC and CTAB was further supported by washing experiments whereby flocculation phenomena only began to decay when the NCC/CTAB nanocomplexes were washed more than 15 times with PBS (data not shown). Other groups have observed a similar interaction between CTAB and negatively charged gold nanoparticles whereby the surfactant binds to the surface of the particle and may cause concentration dependent particle aggregation. ${ }^{24}$ Furthermore, Alkilany et $\mathrm{al}^{25}$ described the partitioning of hydrophobic napthol molecules into surfactant-coated gold nanorods. Interestingly, although gold nanoparticles are used for laser-induced thermal ablation of tumors, these particles have also been modified with hydrophobic polymer chains for the purpose of delivering hydrophobic drugs in a manner similar to CTAB use on NCC described in this study. ${ }^{26}$

The hydrophobic drugs DTX, PTX, and ETOP partitioned strongly into the CTAB domains on NCC using either free drug solutions at low concentrations or micellar solubilized drugs at higher concentrations (Figures 6 and 7). 
These drugs released more slowly from NCC (Figure 9) than the hydrophilic drugs DOX and TET. However, the release profiles were all characterized by a burst phase of release of between $40 \%$ and $75 \%$ of the bound drug over the first 2 days followed by an extremely slow rate of release. These profiles suggest a weakly bound fraction of drug releasing quickly and a strongly bound fraction that released very slowly.

These NCC/CTAB nanocomplexes were shown to associate strongly with KU-7 cancer cells (Figure 11). Because fluorescein was strongly bound within the CTAB coating on the NCC, it was possible to quantitate the cell-bound NCC by measuring the fluorescein emission from the cells. This assay does not differentiate between cell surface association and cellular uptake of NCC but clearly shows that NCC may be used to carry agents (in this case a hydrophobic probe, fluorescein) to cells. This concept is supported by confocal microscopy studies showing a strong fluorescence signal from the cytoplasm of the cancer cells (Figure 12). In these studies, the nuclear and cytoplasmic regions were differentially stained with DAPI (Figure 12B) and fluorescein (Figure 12C), respectively. No fluorescein signal was observed in the location of the nucleus, suggesting the cytoplasm as the location of fluorescein, since surface-bound fluorescein would be observed over the full exposure of the cells. These data indicate cellular uptake of fluorescein but do not differentiate the uptake of free fluorescein from the $\mathrm{NCC} / \mathrm{CTAB} /$ fluorescein nanocomplexes, as it is possible that fluorescein may partition into the hydrophobic cell membrane following cell binding of the nanocomplexes. Since cellular uptake of fluorescein was almost complete by 2 hours and anticancer drugs such as ETOP, PTX, and DTX release occurred over days (Figure 9), it may be assumed that NCC/CTAB/drug nanocomplexes offer a viable and novel method of delivering drugs to cells and may actually deliver these anticancer drugs as controlled release systems (NCC/CTAB/drug nanocomplexes) within cells. These confocal studies also indicate the biocompatibility of the $\mathrm{NCC} / \mathrm{CTAB} /$ nanocomplexes, since cells were intact following incubation with the nanocomplexes for 24 hours. In cytotoxicity studies measuring the release of lactate dehydrogenase (a marker of cytolysis), NCC and NCC-CTAB were found to have no lytic effect at a concentration of $1 \mathrm{mg} / \mathrm{mL}$ (data not shown). However, upon dilution in PBS, lower concentrations of NCC/CTAB (not NCC) were observed to cause some background lysis, indicating that some unbound CTAB might interact directly with the cancer cell membranes. The potential cytotoxic effect of CTAB bound to $\mathrm{NCC}$ is under further investigation.

\section{Conclusion}

In these studies, we investigated the potential of $\mathrm{NCC}$ as a drug delivery excipient. It was demonstrated that NCC was capable of binding significant quantities of the ionizable water soluble antibiotics tetracycline and doxorubicin. These hydrophilic drugs were rapidly released with complete release in 1 day. It was shown that the surface of NCC could be modified by binding the cationic surfactant $\mathrm{CTAB}$, resulting in a concentration dependent increase in the zeta potential of the NCC crystallites. CTAB-coated NCC was shown to bind significant quantities of the nonionized hydrophobic anticancer agents docetaxel, paclitaxel, and etoposide and release these drugs in a controlled manner over several days. The NCC/CTAB nanocomplexes bound to KU-7 bladder cancer cells and demonstrated efficient delivery of a hydrophobic fluorescent probe fluoroscein to the cytoplasm of these cells. Overall, these studies have established the potential of NCC as a drug delivery excipient for use alone or in conjunction with other formulations.

\section{Acknowledgments/disclosure}

The authors gratefully acknowledge financial support from FPInnovations. JKJ, KL, BZW, LY, and HMB are employees of the University of British Columbia and report no conflicts of interest in this work. WYH is an employee of FPInnovations and an Adjunct Professor with the University of British Columbia.

\section{References}

1. O'Connor RE, Schwartz JB. Drug release mechanism from a microcrystalline cellulose pellet system. Pharm Res. 1993;10(3):356-361.

2. Baumann MD, Kang CE, Stanwick JC, et al. An injectable drug delivery platform for sustained combination therapy. J Control Release. 2009; 138(3):205-213.

3. Bley O, Siepmann J, Bodmeier R. Protection of moisture-sensitive drugs with aqueous polymer coatings: importance of coating and curing conditions. Int J Pharm. 2009;378(1-2):59-65.

4. El-Maradny HA. Modulation of a pulsatile release drug delivery system using different swellable/rupturable materials. Drug Deliv. 2007;14(8): 539-546.

5. Gomez-Carracedo A, Souto C, Martinez-Pacheco R, Concheiro A, Gomez-Amoza JL. Microstructural and drug release properties of oven-dried and of slowly or fast frozen freeze-dried MCC-Carbopol pellets. Eur J Pharm Sci. 2007;67(1):236-245.

6. Javadzadeh Y, Shariati H, Movahhed-Danesh E, Nokhodchi A. Effect of some commercial grades of microcrystalline cellulose on flowability, compressibility, and dissolution profile of piroxicam liquisolid compacts. Drug Dev Ind Pharm. 2009;35(2):243-251.

7. Khan GM, Zhu JB. Studies on drug release kinetics from ibuprofencarbomer hydrophilic matrix tablets: influence of co-excipients on release rate of the drug. J Control Release. 1999;57(2):197-203.

8. Knight PE, Podczeck F, Newton JM. The rheological properties of modified microcrystalline cellulose containing high levels of model drugs. J Pharm Sci. 2009;98(6):2160-2169.

9. Kranz H, Jurgens K, Pinier M, Siepmann J. Drug release from MCC- and carrageenan-based pellets: experiment and theory. Eur $J$ Pharm Biopharm. 2009;73(2):302-309. 
10. Podczeck F, Knight PE, Newton JM. The evaluation of modified microcrystalline cellulose for the preparation of pellets with high drug loading by extrusion/spheronization. Int J Pharm. 2008;350(1-2): $145-154$.

11. Watanabe Y, Mukai B, Kawamura K, et al. Preparation and evaluation of press-coated aminophylline tablet using crystalline cellulose and polyethylene glycol in the outer shell for timed-release dosage forms. Yakuga Zasshi. 2002;122(2):157-162.

12. Abdalla A, Klein S, Mader K. A new self-emulsifying drug delivery system (SEDDS) for poorly soluble drugs: characterization, dissolution, in vitro digestion and incorporation into solid pellets. Eur J Pharm Sci. 2008;35(5):457-464.

13. Vinatier C, Gauthier O, Fatimi A, et al. An injectable cellulose-based hydrogel for the transfer of autologous nasal chondrocytes in articular cartilage defects. Biotechnol Bioeng. 2009;102(4):1259-1267.

14. Guo X, Chang RK, Hussain MA. Ion-exchange resins as drug delivery carriers. J Pharm Sci. 2009;98(11):3886-3902.

15. Springate CM, Jackson JK, Gleave ME, Burt HM. Efficacy of an intratumoral controlled release formulation of clusterin antisense oligonucleotide complexed with chitosan containing paclitaxel or docetaxel in prostate cancer xenograft models. Cancer Chemother Pharmacol. 2005;56(3):239-247.

16. Springate CM, Jackson JK, Gleave ME, Burt HM. Clusterin antisense complexed with chitosan for controlled intratumoral delivery. Int J Pharm. 2008;350(1-2):53-64.

17. Hamad W. On the development and applications of cellulosic nanofibrillar and nanocrystalline materials. Can J Chem Eng. 2006;84: 513-519.
18. Habibi Y, Lucia LA, Rojas OJ. Cellulose nanocrystals: chemistry, self-assembly, and applications. Chem Rev. 2010;110(6):3479-3500.

19. Shaikh S, Birdi A, Qutubuddin S, Lakatosh E, Baskaran H. Controlled release in transdermal pressure sensitive adhesives using organosilicate nanocomposites. Ann Biomed Eng. 2007;35(12):2130-2137.

20. Lönnberg H, Fogelströma L, Samir MASA, et al. Surface grafting of microfibrillated cellulose with poly( $\varepsilon$-caprolactone) - Synthesis and characterization Eur Polym J. 2008;44(9):2991-2997.

21. Zhang X, Jackson JK, Burt HM. Development of amphiphilic diblock copolymers as micellar carriers of taxol. Int J Pharm. 1996;132(1-2): 195-206.

22. Pan J, Hamad W, Straus SK. Parameters affecting the chiral nematic phase of nanocrystalline cellulose films. Macromolecules. 2010;43: 3851-3858.

23. Hamad W, Hu TQ. Structure-property-yield interrelationships in nanocrystalline cellulose extraction. Can J Chem Eng. 2010;88(3): 392-402.

24. Yang Y, Matsubara S, Nogami M, Shi J, Huang W. One-dimensional self-assembly of gold nanoparticles for tunable surface plasmon resonance properties Nanotechnology. 2006;17(11):2821.

25. Alkilany A, Frey R, Ferry J, Murphy C. Gold nanorods as nanoadmicelles: 1-naphthol partitioning into a nanorod-bound surfactant bilayer. Langmuir. 2008;24(18):10235-10239.

26. Kim J-H, Lee TR. Discrete thermally responsive hydrogel-coated gold nanoparticles for use as drug-delivery vehicles. Drug Develop Res. 2006;67:61-69.
International Journal of Nanomedicine

\section{Publish your work in this journal}

The International Journal of Nanomedicine is an international, peerreviewed journal focusing on the application of nanotechnology in diagnostics, therapeutics, and drug delivery systems throughout the biomedical field. This journal is indexed on PubMed Central, MedLine, CAS, SciSearch $\AA$, Current Contents ${ }^{\circledR} /$ Clinical Medicine,

\section{Dovepress}

Journal Citation Reports/Science Edition, EMBase, Scopus and the Elsevier Bibliographic databases. The manuscript management system is completely online and includes a very quick and fair peer-review system, which is all easy to use. Visit http://www.dovepress.com/ testimonials.php to read real quotes from published authors. 